\title{
SXS and XPS Study of the Adsorption and Desorption of Te on GaAs (100)
}

\author{
J.C. González, W.N. Rodrigues, C.M. Silva, M.V.B. Moreira, A.G. de Oliveira, \\ Departamento de Física - ICEx - UFMG, CP 702, 30123-970, Belo Horizonte - Brazil
}

M. Abbate, and F.C. Vicentin

LNLS, CP 6192, 13083-970, Campinas - Brazil

Received February, 1998

\begin{abstract}
The adsorption and desorption of Te on GaAs (100) has been investigated using Soft X-ray Spectroscopy (SXS) and X-ray Photoelectron Spectroscopy (XPS). The SXS measurements have been done at the Brazilian synchrotron facility LNLS, and the XPS study was performed using laboratory sources. The samples were MBE GaAs (100) layers covered in-situ by Te from an effusion cell. Two families of samples were studied: samples analyzed in-situ, and samples that were exposed to air for at least one week. The desorption of Te of the non-oxidized samples indicates that traces of $\mathrm{Te}$ remains in the surfaces for temperatures as high as $6800^{\circ} \mathrm{C}$. However, for the case of the oxidized samples, the Te film sublimates easily at around of $250^{\circ} \mathrm{C}$, leaving a clean GaAs surface. It seems that the oxidized Te film works as a suitable protection for epitaxial GaAs layers.
\end{abstract}

The study of adsorption and desorption of Te on GaAs (100) is very important since Te can be used in the Surfactant Controlled Molecular Beam Epitaxy of InAs on GaAs [1]. In this work we investigated the adsorption of Te on the surface of MBE grown GaAs (100) using an in-situ effusion cell for the deposition of Te. The evolution of the adsorption of Te was monitored using in-situ XPS. The study of the desorption of Te was carried out in two different kind of samples, namely non-oxidized samples and samples exposed to air for at least one week. The non-oxidized samples were studied in-situ after the deposition of Te. The procedure used was to anneal the sample at a known temperature and to measure the XPS spectra, once the sample was at room temperature. RHEED experiments were carried out in this kind of sample, measuring the intensity of diffracted beams as a function of annealing temperature. The air-exposed samples were studied using the same procedure, but SXS experiments were carried out instead of RHEED.

The integrated intensity of Te as a function of the deposition time is shown in Fig.1. This process of adsorption of Te is characterized by two different deposition rates. In the beginning the deposition rate is 0.16 $\AA / \mathrm{s}$ or $0.043 \mathrm{ML} / \mathrm{s}$, considering that one monolayer of
Te correspond to $3.72 \AA$ [2]. This rate is constant during the first $6 \AA$, but changes to $0.03 \AA / \mathrm{s}$ after $6 \AA$ of Te. This change in the deposition rate of Te suggests a change in the sticking coefficient of Te to the surface, at that critical thickness.

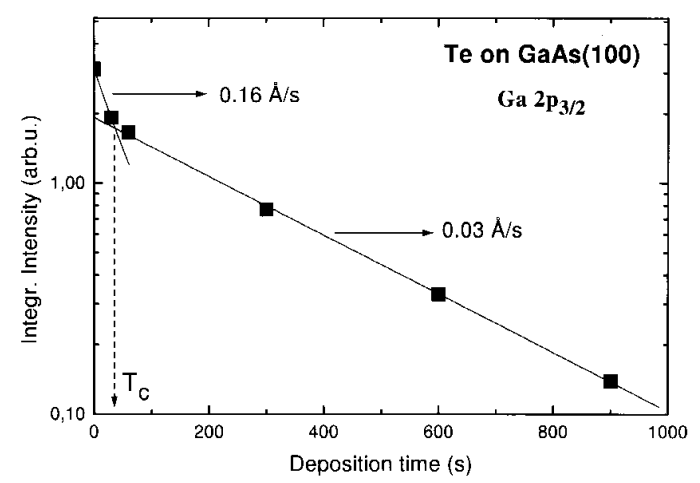

Figure 1. Evolution of the Ga $2 \mathrm{p}_{3 / 2}$ XPS peak with the deposition time of Te. Two deposition rates are observed. At $T_{c}$ the $T e$ film is $6 \AA$ thick, and the growth rate changes abruptly from $0.16 \AA / \mathrm{s}$ to $0.03 \AA / \mathrm{s}$.

The in-situ studied desorption of Te represented in Fig. 2a and 2b, show interesting results not yet fully understood. The RHEED intensity evolution shows different structures, which may be related to the evaporation of successive layers of Te. The XPS measurements indicate that as the temperature increases first 
the Te signal decreases, with the expected increment in the $\mathrm{Ga}$ and As peaks. However around $580{ }^{\circ} \mathrm{C}$ the Ga peaks starts to reduce in intensity, the As peaks increases strongly and the Te peak stabilizes until 600 ${ }^{\circ} \mathrm{C}$. This can be due to preferential As migration to the surface. This behavior is observed until $630^{\circ} \mathrm{C}$, when Te almost completely disappears. After $630^{\circ} \mathrm{C}$ the Ga peak increases, and the As signal reduces, indicating a Ga-enriching process at the surface. The annealing continues until $680^{\circ} \mathrm{C}$ and the surface remains stable. This is one remarkable result since usually at so high temperatures GaAs degrades under UHV.
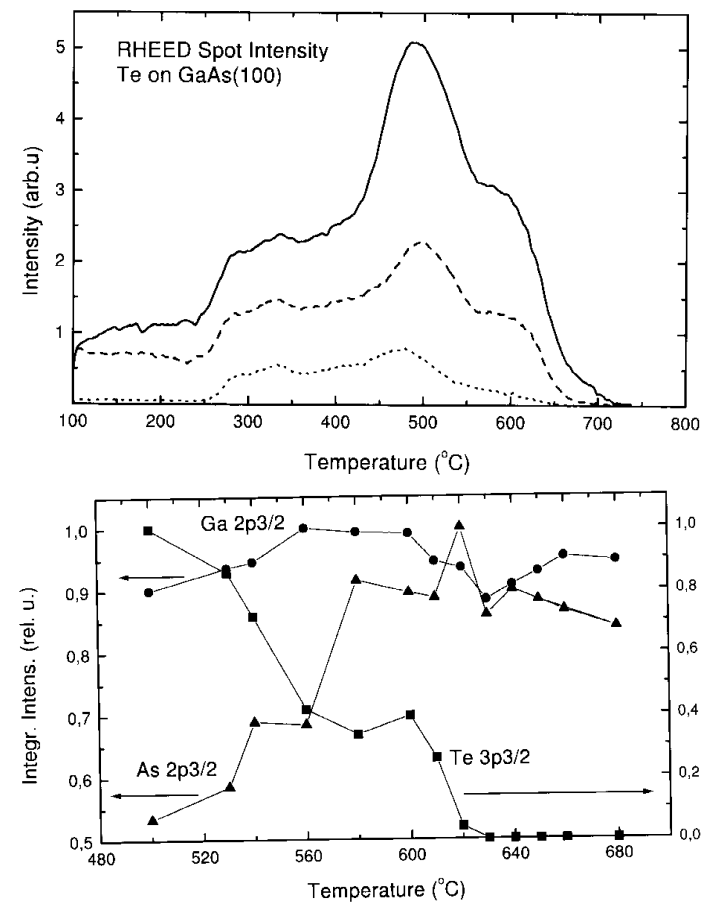

Figure 2. Te desorption from GaAs (100) surface: a) RHEED intensity as function of the annealing temperature, for three different diffracted spots, b) evolution of the peaks of $\mathrm{Ga}$, As and Te as a function of the annealing temperature.

Fig. 3 shows the XPS Ga $2 p_{1 / 2}$ and Ga $2 p_{3 / 2}$ peaks, of the air-exposed samples, for different annealing temperatures. An abruptly change is observed in the intensity of these peaks when the temperature increases from $200^{\circ} \mathrm{C}$ to $250^{\circ} \mathrm{C}$. In Fig. 4 a the evolution of the peaks of Te, Ga and As is shown. We can conclude that these changes are due to the Te evaporation at this range of temperature. Desorption of Te in the air-exposed samples, shows that at $250{ }^{\circ} \mathrm{C}$ the concentration of Te in the surface is not more detectable by XPS. This is corroborated by the SXS measurements, shown in Fig. $4 \mathrm{~b}$. These results show that the oxidation of the cap layer of Te creates a volatile layer, which can be useful in obtaining clean GaAs surfaces.

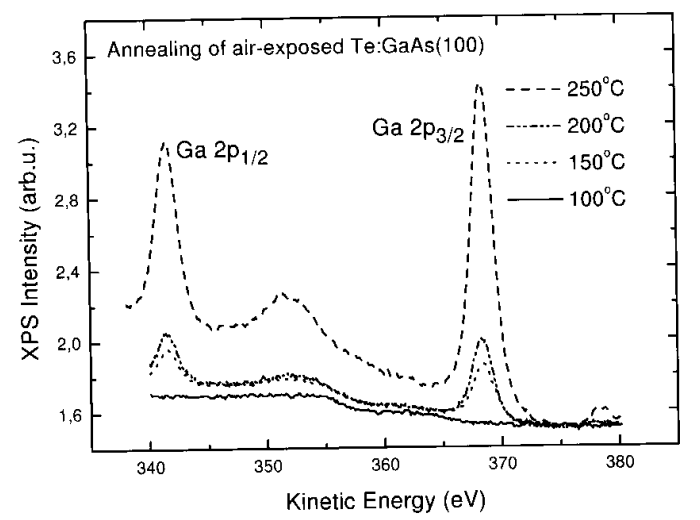

Figure 3. Evolution of the Ga $2 \mathrm{p}$ peaks as a function of the annealing temperature, for air exposed Te:GaAs samples.
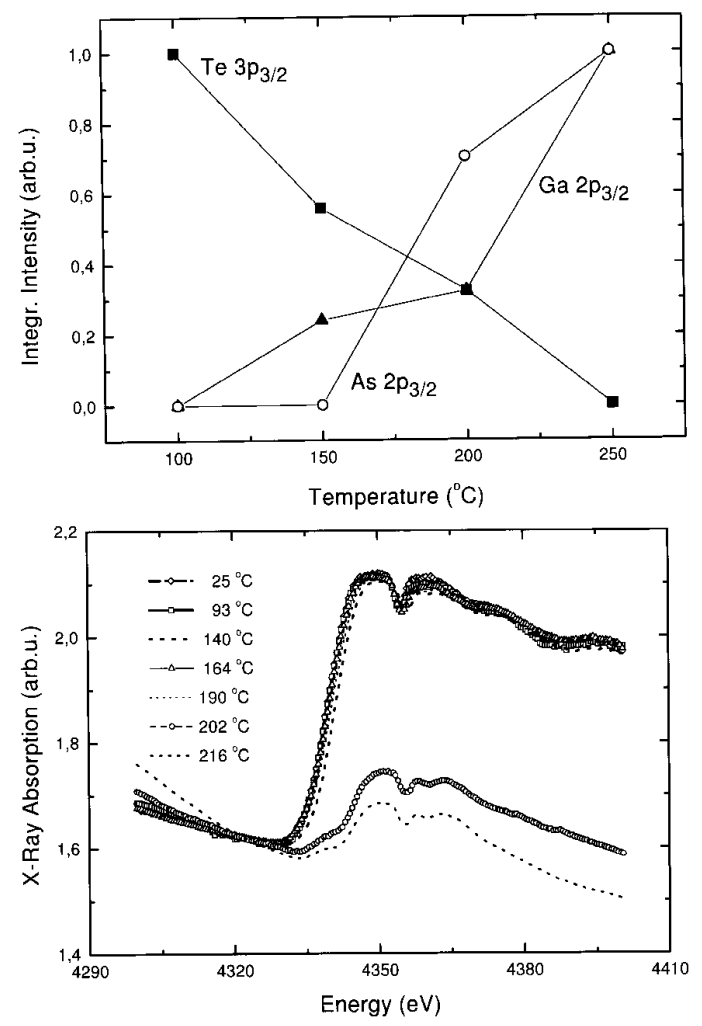

Figure 4. Desorption of Te in air exposed samples: a) evolution of the peaks of $\mathrm{Ga}$, Te, As as a function of the annealing temperature, b) evolution of the X-ray absorption at the Te $\mathrm{K}$ edge, at different annealing temperatures.

As a conclusion, we can say that the deposition rate of Te depends of the thickness of the Te layer. The study of desorption of Te in non-oxidized samples shows that $\mathrm{Te}$ is detectable in the surface until $620^{\circ} \mathrm{C}$. However the unusual stability of the GaAs surface at temperatures so high as $680^{\circ} \mathrm{C}$, as demonstrated in this work, suggest that minor traces of Te are still present at the highest temperature attained. 
The desorption studies in the air exposed samples show that Te sublimes at $\sim 250{ }^{\circ} \mathrm{C}$ leaving a clean GaAs surface. This oxidized Te forms a protective and volatile layer for the GaAs surface.

\section{Acknowledgements}

The authors would like to thank the agencies CNPq, CLAF, FINEP, and FAPEMIG for their support.

\section{References}

1. W.N. Rodrigues, V.H. Etgens, M. SauvageSimkin, G. Rossi, F. Sirotti, R. Pinchaux, and F. Rochet, Solid State Comm., 95, 873 (1995).

2. A.C. Ferraz and R. Claudino da Silva, Surf. Sci. 352-354, 379 (1996). 